\title{
Staphylococcus hominis: experiencia de contaminación en el pro- ceso de vacunación en Chiapas
}

La vacunación es un componente esencial del derecho humano a la salud. Se estima que, gracias a ella, cada año se previenen alrededor de 2,5 millones de fallecimientos a nivel mundial. México, es uno de los países a favor del bienestar de la niñez, por tal motivo desde 1991 surge, como compromiso nacional, la implementación del Programa de Vacunación Universal. Este programa pretende, además de mejorar la supervivencia infantil, promover, proteger y cuidar la salud de todos los niños del país, a través de acciones de vacunación gratuita, así como promoción y educación para la salud y participación comunitaria como mecanismos de apoyo para la prevención de las enfermedades que no se evitan vacunandose.

El Programa Mexicano de Vacunación (PMV), cuenta con uno de los esquemas más completos a nivel mundial, con un total de 14 vacunas; anualmente, se aplican alrededor de 80 millones de dosis seguras y eficaces (1). Gracias a este programa el país se encuentra libre de enfermedades como la poliomielitis, el sarampión o la rubéola y ha contribuido a reducir la mortalidad infantil. El esquema actual de vacunación protege contra formas graves de tuberculosis, hepatitis B, difteria, tos ferina, tétanos, poliomielitis, enfermedades por influenza tipo B, sarampión, rubéola y parotiditis, enfermedad diarreica por rotavirus, infecciones invasivas por neumococo y virus de la influenza (2).

La vacuna de la BCG y hepatitis B son aplicadas poco después del nacimiento, $\mathrm{y}$, posteriormente, en el transcuros de los primeros dos meses de vida se aplica la pentavalente acelular (el toxoide diftérico, el toxoide tetánico, el toxoide pertúsico, los virus de la poliomielitis inactivados tipos I, II y III; y una proteína de la bacteria Haemophilus influenzae tipo b), hepatitis $\mathrm{B}$, rotavirus y neumococo conjugada (3).

Para garantizar la seguridad, calidad y eficacia de las vacunas, en México se cuenta con la Comisión Federal para la Protección contra Riesgos Sanitarios (COFEPRIS), reconocida en 2012 como autoridad reguladora nacional de referencia regional de medicamentos y productos biológicos por la Organización Panamericana de la Salud (OPS) y en 2014 como Agencia Funcional en materia de vacunas de la Organización Mundial de la Salud (OMS). Es importante comentar que sólo algunos organismos, en 28 países en el mundo, cuentan con esta denominación. De acuerdo con los más altos estándares internacionales, la evaluación de todos los productos biológicos realizada en la COFEPRIS comprende tres etapas: 1) La evaluación técnica-científica de la documentación de producción de las vacunas para la obtención del registro sanitario, en el que se verifica que todos los procesos de producción, desde las materias primas hasta el producto terminado, se lleven a cabo en establecimientos que cuenten con el certificado de buenas prácticas de manufactura. Adicionalmente, se analizan las estabilidades y los estudios clínicos que certifican la seguridad y eficacia de las vacunas; 2) la realización de las pruebas que reportan en el certificado de análisis del producto para cada lote. Los resultados de estas pruebas se comparan con los estándares establecidos en la Farmacopea de los Estados Unidos Mexicanos (FEUM); 3) por último, para autorizar la liberación de los lotes, la COFEPRIS realiza la evaluación analítica sobre el producto terminado, es decir, realiza las pruebas indicadas en la FEUM y verifica que los resultados 
Dzul-Rosado et al.

obtenidos sean concordantes con el certificado de análisis de producto y que se encuentren dentro de las especificaciones. Mediante este proceso, la COFEPRIS evalúa, la calidad de las materias primas utilizadas para la producción de las vacunas, el proceso de producción, así como transporte y almacenamiento de los biológicos. Por lo anterior, cuando una vacuna es aplicada, ésta ya ha pasado tres filtros de seguridad garantizando que no constituye un riesgo para la población (1).

Lo sucedido el pasado Mayo, tras la vacunación de bebés en dos comunidades indígenas de Chiapas donde dos de ellos perdieron la vida y 37 más resultaron hospitalizados, es indicativo que posibles causa ajenas a este riguroso proceso pudieran haberse presentado.

En las comunidades indígenas, La Pimienta y San José, del Municipio de Simojovel en el estado de Chiapas, después de haber realizado la vacunación de menores contra hepatitis B, éstos comenzaron a presentar signos y síntomas clínicos que los llevó a presentar un cuadro clínico grave y complejo, caracterizado por crisis convulsivas, culminando con el deceso de dos neonatos y afectación importante en otros. El primer reporte, dado a través de las redes sociales de Simojovel, expuso el hecho causando pánico entre la población de estas comunidades integradas, principalmente, por indígenas de la etnia tzotzil quienes viven en marginación extrema. Es importante comentar que la vacunación ha demostrado ser una de las estrategias de salud pública con mayor efectividad disminuyendo la presencia de enfermedades inmunoprevenibles; sin embargo, hay que considerar que todo medicamento, incluidas las vacunas, pueden causar reacciones adversas leves, moderadas o graves. Con lo anterior, se suspendió la jornada de vacunación, ya que cabía la posibilidad de que de que las afectaciones fueran provocadas por la aplicación.

Los primeros resultados de los cultivos microbiológicos indicaron la presencia de contaminación externa por bacterias. Considerando que los cuadros clínicos que presentaron los neonatos estuvieron caracterizados por alteraciones del sistema nervioso y respiratorio, todo estaría indicando compatibilidad con la presencia de algún microorganismo. Es importante comentar la prontitud con la que fue conformado un equipo multidisciplinario para salvaguardar la salud de los niños, dividiéndose en dos grupos, uno, con el objetivo de apoyar económicamente a las familias y el otro, liderado por la COFEPRIS y la SSA. Después que las investigaciones dieron resultado, COFEPRIS determinó que las vacunas no presentaban anomalías y que no habían perdido la cadena de frío, y que, probablemente, el problema ocurrido en Chiapas haya sido focalizado, ya que estos lotes tenían decenas de miles de dosis aplicadas desde octubre hasta la fecha, sin que existiera algún caso de reacción. En cuanto a los estudios microbiológicos, los resultados confirmaron que la bacteria implicada en los cuadros infecciosos fue Staphylococcus hominis.

El Staphylococcus hominis (S. hominis), es un miembro de las bacterias coagulasa negativa del género Staphylococcus; se encuentra, comúnmente, como un comensal inocuo en la piel humana y animal. Sin embargo, al igual que muchos otros estafilococos coagulasa negativa, S. hominis pueden, ocasionalmente, causar una infección en pacientes con sistemas inmunológicos comprometidos; tiende a colonizar en áreas con numerosas glándulas apocrinas, como las axilas y la región púbica. Se ha reportado que $S$. hominis se presenta en $22 \%$ del total de especies de estafilococos recuperados de los individuos. La mayoría de las cepas colonizan en la piel durante períodos de tiempo relativamente cortos en comparación con otras especies de Staphylococcus (4). En la literatura se ha descrito subespecies de $S$. hominis, como S. hominis subsp. novobiosepticus (SHN), este

\section{Revista Biomédica}


Vacunación en Chiapas

nombre deriva de la combinación de novobio, perteneciente a la propiedad de la resistencia novobiocina y septicus, perteneciente a la capacidad de causar sepsis, y ha sido descrito como un importante patógeno nosocomial en neonatos (5).

Así mismo los estudios moleculares realizados demostraron, también, que el $S$. hominis aislado en los diferentes pacientes fue el mismo. Es decir, la bacteria provino de una sola fuente de contaminación. El análisis de la COFEPRIS indicó que la contaminación con esta bacteria ocurrió durante el procedimiento de manejo y aplicación de la vacuna. Lo anterior nos hace pensar que, si bien la cadena de procedimientos previos al ingreso de una vacuna no se vio alterada, hay que considerar igualmente importantes todos los componentes presentes alrededor del proceso de la aplicación como la trasmisión cruzada que resulta de la ruptura de alguna o algunas de las técnicas habituales, como por ejemplo lavado de manos, circulación excesiva de personal, personal insuficiente o malas técnicas de curación.

Estos resultados preliminares, obligan a fortalecer la importancia de los cuidados que implica el proceso de vacunación tanto en la comunidad médica y en la población en general, con el fin de garantizar el principal objetivo del programa de vacunación universal, proteger contra enfermedades que son prevenibles a través de la aplicación de vacunas.

Dra. Karla Dzul Rosado

Dr. Cesar Lugo Caballero

Dr. Jorge Zavala Castro

MIBB Fernando I. Puerto

Centro de Investigaciones Regionales-HN

Universidad Autónoma de Yucatán

\section{REFERENCIAS}

1. Secretaría de Salud. Comunicado de prensa número 266. Disponible en : http://www.salud.gob.mx/ ssa_app/noticias/datos/2015-05-14_7562.html Fecha de acceso: 02 de Junio del 2015

2. Ramírez A. Importancia de la Vacunación. Disponible en : http://www.voluntariado.salud.gob.mx/movil/ noticias/vacunas/importanciavacunas.html. Fecha de acceso: 02 de Junio del 2015.

3. Secretaría de salud, CENSIA. Esquema de vacunación. Disponible en: http://www.censia.salud. gob.mx/contenidos/vacunas/esquema.html. Fecha de aceso: 02 de Junio del 2015

4. Cercenado E, García-Leoni ME, Díaz MD, Sánchez-Carrillo $C$, Catalán $P$, De Quirós JC, Bouza E. Emergence of teicoplanin-resistant coagulase-negative staphylococci. J Clin Microbiol. 1996 jul; 34(7):1765-8.

5. Chaves F, García-Alvarez M, Sanz F, Alba C, Otero JR. Nosocomial spread of a Staphylococcus hominis subsp. novobiosepticus strain causing sepsis in a neonatal intensive care unit.. J Clin Microbiol. 2005 sep; 43(9):4877-9. 